\title{
Raman Crosstalk in Fiber-Optic Hybrid CATV Systems With Wide Channel Separations
}

\author{
Feng Tian, Rongqing Hui, Barry Colella, and David Bowler
}

\begin{abstract}
The transfer function of nonlinear Raman crosstalk in fiber-optic systems is investigated both experimentally and analytically. When the channel separation is wide enough, the frequency response of the Raman crosstalk can be significantly changed by the effect of polarization-mode dispersion. Matched polarization state between the two channels at the fiber input does not necessarily ensure the worst case crosstalk for all frequency components. The implication of this effect in design of fiber-optic hybrid CATV systems is discussed.

http://www.corning.com/opticalfiber/products_services/product _catalog/smf-2
\end{abstract}

Index Terms-Cable television (CATV), crosstalk, nonlinear optics, optical fiber communications, Raman scattering.

\section{INTRODUCTION}

$\mathbf{N}$ ONLINEAR Raman crosstalk in fiber-optic WDM systems has been a subject of intensive study for the last few years [1]-[3]. Fiber-optic analog systems such as cable television (CATV) are especially susceptible to the nonlinear crosstalk because of the stringent carrier-to-noise-ratio (CNR) requirement. Detailed analysis on interchannel Raman crosstalk in CATV systems has been reported in [4], where the wavelength spacing between channels is typically less than $10 \mathrm{~nm}$. In these cases, the strongest crosstalk most likely happens when all the wavelength channels are copolarized at the input of the fiber system. Indeed this assumption has been used to find the worst case crosstalk. In recent years, passive optical network (PON) technology has been introduced to bring broadband data, voice and video services to residential and business users. In such a network, a 1550-nm wavelength video channel travels downstream with a 1490 -nm wavelength data channel on a single fiber. Although the video channel carries a much stronger optical power when compared to the 1490-nm data channel, the performance of the analog CATV channels may still be significantly degraded by the presence of the data channel due to nonlinear Raman crosstalk if the system is not designed properly [5]. In this letter, we will demonstrate that due to the wide wavelength separation between data and video channels, their polarization states may walkoff rapidly because of random polarization mode dispersion (PMD) along the fiber. Therefore, the frequency transfer function of Raman crosstalk is very sensitive to the signal polarization states and

Manuscript received June 24, 2003; revised August 12, 2003.

F. Tian, B. Colella, and D. Bowler are with the Quantum Bridge Communications, Andover, MA 01810 USA.

R. Hui is with the Department of Electrical Engineering and Computer Science, University of Kansas, Lawrence, KS 66044 USA.

Digital Object Identifier 10.1109/LPT.2003.818899 copolarization at the fiber input does not necessarily imply the worst case nonlinear crosstalk.

\section{EXPERIMENT}

In order to investigate the effect of nonlinear Raman crosstalk between two channels with wide wavelength separation, laboratory measurements have been conducted. Two optical transmitters are used with emission wavelengths of 1550 and $1490 \mathrm{~nm}$, respectively. These two channels are combined by a wavelength multiplexer and transmitted through a standard single-mode fiber (SMF). The polarization state of each transmitter is individually adjusted by a polarization controller before the WDM combiner. The 1550-nm transmitter is operated at CW and the 1490-nm transmitter is intensity modulated by a swept frequency RF source provided by an RF network analyzer. The optical signal at the end of the transmission fiber is filtered by an optical bandpass filter which selects the 1550-nm channel and rejects the 1490-nm channel. Due to the nonlinear Raman crosstalk, the modulated frequency component is transferred from the 1490-nm channel to the 1550-nm channel. A wideband optical receiver is used to detect the optical signal before sending it to the receiver port of the RF network analyzer. By sweeping the modulation frequency, the transfer function of the nonlinear Raman crosstalk is obtained through the measurement of the S21 parameter in the Network analyzer. A normalization is necessary to generalize the results since the absolute level of the transfer function depends on the gain of electrical amplifier as well as the responsivity of the photodetector. To this end, we define the normalized transfer function as $T(f)=\delta P_{1}(f) / P_{10} \cdot \delta P_{1}(f)$ and $P_{10}$ are the electrical power spectral density of Raman crosstalk and the average electrical power, respectively, of the 1550-nm channel after photodiode.

Fig. 1 shows the normalized transfer functions of nonlinear Raman crosstalk measured using $10 \mathrm{~km}$ of standard SMF (SMF-28). The optical power levels were $0 \mathrm{dBm}$ and $17 \mathrm{dBm}$ for the 1490- and 1550-nm channels, respectively. The transfer function describes the detailed frequency-dependent efficiency and reveals the nature of the Raman crosstalk, which is a powerful tool in system performance evaluation. Two measured transfer functions in Fig. 1 illustrate the dramatic change in the crosstalk transfer function which was obtained only by changing the signal polarization states and the PSPs of the transmission fiber through the adjustment of the polarization controllers. Although it is well known that the efficiency of Raman crosstalk depends on the relative polarization states of the two wavelength channels [4], [5], we found that the 


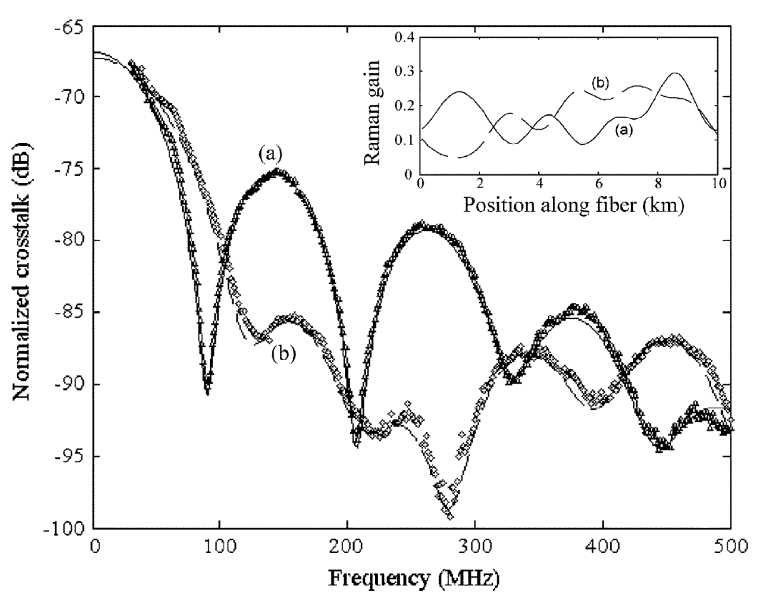

Fig. 1. Measured (opened triangles and diamonds) and calculated (solid and dashed lines) transfer functions of nonlinear Raman crosstalk in the same system but with different polarization conditions. Inset: Profiles of Raman gain coefficients along the fiber used in the calculation to fit the measured transfer functions (a) and (b), respectively.

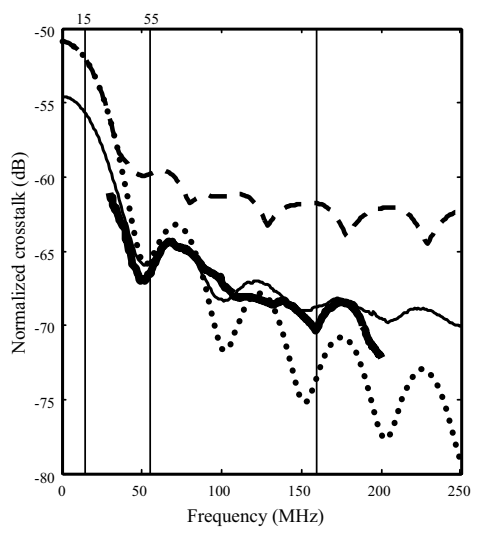

(A)

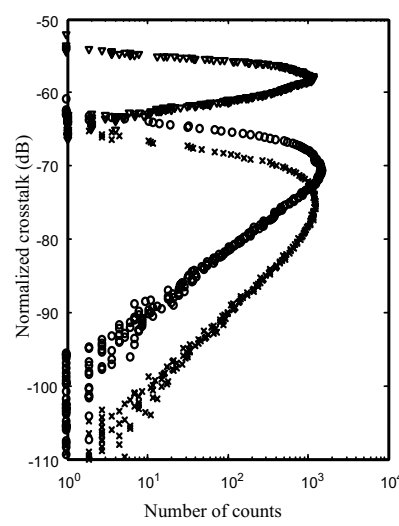

(B)
Fig. 2. (a) Calculated absolute worst case (dashed line) and $99 \%$ worst case (solid line) Raman crosstalk in a 20-km fiber system. The bold line represents the measured worst case, which were measured using a maximum-holding on the network analyzer. Dotted line: calculated Raman crosstalk assuming perfect signal polarization alignment along the fiber. (b) Calculated statistica distribution of Raman crosstalk at $15-\mathrm{MHz}$ (triangles), 55-MHz (open circles) and $160-\mathrm{MHz}$ (open triangles) modulation frequencies. $10^{5}$ calculations were made at each wavelength.

frequency dependency of this crosstalk was very sensitive to the polarization states of the input optical signals as well as the fiber PSP. A direct consequence of this phenomenon is that the system performance will vary over time due to the fluctuation of the fiber PSPs.

In order to evaluate the worst case transfer function of the crosstalk, a maximum-hold function on the network analyzer can be used while varying the fiber PSPs and the input signal polarization states. The result is shown in Fig. 2 (by the bold line). This measurement was performed on a system with $20 \mathrm{~km}$ of SMF-28. Although the instantaneous transfer functions vary with time, the maximum-hold values are deterministic, which indicates the existence of a worst case limit.

\section{THEORY AND DISCUSSION}

In previous studies, Raman gain coefficient $g$ has been considered as a constant along the fiber. In practice, Raman gain coefficient is related to the relative polarization states of the two channels involved in the Raman crosstalk process. It is generally accepted that Raman gain is maximum when the two channels have the same polarization states, while no Raman crosstalk is expected when the two channels are orthogonally polarized. It is also well known that due to PMD in the transmission fiber, different wavelength channels will experience different polarization rotation during the propagation along the fiber. In fact, the differential group delay (DGD) $\Delta \tau$ is directly related to the angular walfoff $\Delta \theta$ of the two channels on the poincare sphere [6]: $\Delta \tau=-\lambda_{0}^{2} \Delta \theta /(2 \pi c \Delta \lambda)$, where $\Delta \lambda$ is the wavelength separation between two channels.

A direct consequence of this polarization walkoff is the reduction of Raman gain coefficient $g$ when the lightwaves propagate along the fiber. Most importantly, since the polarization walkoff is on the order of multiple $\pi$ 's in PON systems with wide wavelength channel separation, the Raman gain is position-dependent along the fiber. Because of the temporal walkoff between the two channels due to chromatic dispersion, nonlinear crosstalk originated at different locations along the fiber will likely contribute to different frequency spectra in the overall transfer function. Although the effect of PMD and thus the angular walkoff of the polarization states between the two channels is random in nature, we can describe this phenomenon by representing the Raman gain $g(z)$ along the fiber at any given time as Fourier series,

$$
g(z)=\frac{g_{0}}{2}\left[1+\sum_{n=0}^{N} k_{n} \cos \left(\frac{2 n \pi}{L} z+\theta_{n}\right)\right]
$$

where $g_{0}$ is the peak Raman gain which occurs when the two channels are copolarized. $n$ quantifies the rate of rotation of the relative polarization states along the fiber. Higher values of $n$ represent a higher PMD in the fiber. $\theta_{n}$ is an initial phase angle, together with coefficient $k_{n}$ indicating the polarization state mismatch at the fiber input.

With this assumption of location-dependent Raman gain coefficient, the overall Raman crosstalk at the end of a fiber of length $L$ calculated by the well-known coupled-mode equations [4] and the result can be expressed in a closed form. The RF power of the Raman crosstalk, normalized by the average signal power at channel 1, can be found as (2), at the top of the next page. Equation (2) is a general expression of frequency-dependent Raman crosstalk, it takes into account the variation of Raman gain coefficient along the fiber caused by channel polarization walkoff. $\alpha$ is the fiber attenuation and $d_{12}=1 / V_{1}-1 / V_{2}$ is the relative delay between the two channels, with $V_{1}$ and $V_{2}$ the group delay of the two channels. $m$ is the modulation index. This normalized crosstalk is proportional to the average input power $I_{20}$ of the interfering channel and peak Raman gain coefficient $g_{0}$ which is a function of the wavelength spacing. By adjusting $k_{n}, \theta_{n}$ and $N$ in (1), arbitrary variations of Raman gain coefficient along the fiber can be produced. As an example, the solid line (a) and the dashed line (b) in Fig. 2 show the calculated transfer functions that best match the measured results shown as open dots. The parameters used in the calculation are $I_{20}=1 \mathrm{~mW}, m=1$, $g_{0}=0.34 / \mathrm{W} / \mathrm{km}, \Delta \lambda=60 \mathrm{~nm}$. To obtain the best match for each curve in Fig. 1, specific profiles of Raman gain coefficient along the fiber had to be assumed as shown by the inset of Fig. 1. The excellent agreement between the theoretical 


$$
P=\frac{1}{2}\left(p^{2}+q^{2}\right)\left(1+e^{-2 \alpha L}-2 e^{-\alpha L} \cos \Omega d_{12} L\right)
$$

where

$$
\begin{aligned}
& p=\frac{g_{0} I_{20} m}{4}\left\{\frac{-2 \Omega d_{12}}{\alpha^{2}+\left(\Omega d_{12}\right)^{2}}-\sum_{n=0}^{N}\left\{k_{n} \frac{\left(\frac{\Omega d_{12}+2 n \pi}{L}\right) \cos \theta_{n}+\alpha \sin \theta_{n}}{\alpha^{2}+\left(\frac{\Omega d_{12}+2 n \pi}{L}\right)^{2}}+k_{n} \frac{\left(\frac{\Omega d_{12}-2 n \pi}{L}\right) \cos \theta_{n}-\alpha \sin \theta_{n}}{\alpha^{2}+\left(\frac{\Omega d_{12}-2 n \pi}{L}\right)^{2}}\right\}\right\} \\
& q=\frac{g_{0} I_{20} m}{4}\left\{\frac{2 \alpha}{\alpha^{2}+\left(\Omega d_{12}\right)^{2}}-\sum_{n=0}^{N}\left\{k_{n} \frac{\left(\frac{\Omega d_{12}+2 n \pi}{L}\right) \sin \theta_{n}-\alpha \cos \theta_{n}}{\alpha^{2}+\left(\frac{\Omega d_{12}+2 n \pi}{L}\right)^{2}}-k_{n} \frac{\left(\frac{\Omega d_{12}-2 n \pi}{L}\right) \sin \theta_{n}+\alpha \cos \theta_{n}}{\alpha^{2}+\left(\frac{\Omega d_{12}-2 n \pi}{L}\right)^{2}}\right\}\right\}
\end{aligned}
$$

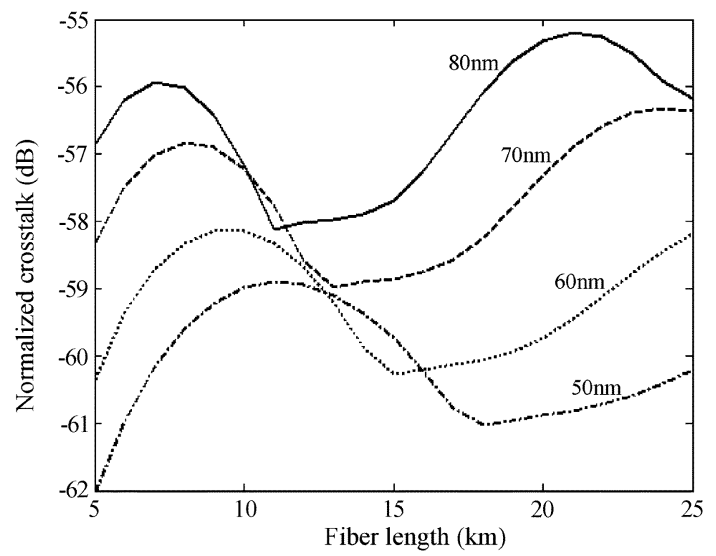

Fig. 3. Calculated worst case Raman crosstalk at $55 \mathrm{MHz}$ versus fiber length for four different wavelength separations of $\Delta \lambda=80 \mathrm{~nm}$ (solid line), $70 \mathrm{~nm}$ (dashed line), $60 \mathrm{~nm}$ (dotted line) and $50 \mathrm{~nm}$ (dashed-dotted line).

calculation and our measurement verifies the hypothesis that the frequency dependency of Raman crosstalk depends on the fiber birefringence and ensures the accuracy of our modeling.

In Fig. 2(a), the dashed line shows the absolute maximum level of Raman crosstalk calculated versus frequency. The solid line in the same figure indicates the $99 \%$ worst case crosstalk level. This was obtained by making $10^{5}$ calculations at each frequency, assuming a uniform distribution for $k_{n} \in(0,1) / \sum_{i=1}^{N} k_{i}$ and $\theta_{n} \in(0,2 \pi), 10$ Fourier terms were used $(N=10)$. This calculated $99 \%$ worst case agrees well with the experimentally measured transfer function using maximum-holding. It was generally accepted that the worst case Raman crosstalk can be predicted by assuming copolarization of the two wavelength channels all along the fiber and no polarization walkoff. The dotted line in Fig. 2(a) was calculated under this condition. Although in low frequencies (up to $35 \mathrm{MHz}$ ) this approximation coincides with the absolute worst case, it underestimates the crosstalk level at high frequencies. As a reference, Fig. 2(b) shows the statistic distributions of Raman crosstalk calculated at 15, 55, and $160 \mathrm{MHz}$, respectively. At low frequencies, the width of the statistic distribution is relatively narrow and this width becomes widened with the increase of frequency. For $20 \mathrm{~km}$ of SMF with dispersion parameter $16.5 \mathrm{ps} / \mathrm{nm} / \mathrm{km}$, the accumulated chromatic dispersion is $330 \mathrm{ps} / \mathrm{nm}$. The group delay difference between two wavelength channels $60 \mathrm{~nm}$ apart is approximately $19.8 \mathrm{~ns}$. This group delay difference is much shorter than the RF period for a modulation frequency at $15 \mathrm{MHz}$, and therefore interference effect is not significant. At a modulation frequency of $55 \mathrm{MHz}$, the RF period is approximately $18 \mathrm{~ns}$, which is on the same order of the group delay difference, both constructive and destructive interference may occur depending on the polarization walkoff between the two channels, making the crosstalk level less predictable.

It was predicted that a global worst case Raman crosstalk happened with a fiber length of approximately $10 \mathrm{~km}$ assuming aligned polarization states [5], where the worst case crosstalk was found always at the lower frequency limit of CATV band $(55 \mathrm{MHz})$. In order to make sure this global worst case still holds when random polarization walkoff is considered, we calculated Raman crosstalk at 55-MHz RF frequency versus fiber length for several different channel spacing, as shown in Fig. 3. At the channel spacing lower than $70 \mathrm{~nm}$, the worst case crosstalk does happen at fiber length of approximately $10 \mathrm{~km}$ as predicted by [5]. However, with wider channel spacing ${ }^{1}$, the worst case crosstalk may move to longer fiber lengths and these worst cases do not correspond to copolarization of the two channels. To obtain results in Fig. 3, typical fiber parameters were used [7]: dispersion slope $S_{0}=0.086 \mathrm{ps} /\left(\mathrm{nm}^{2} \cdot \mathrm{km}\right)$ and zero dispersion wavelength $\lambda_{0}=1313 \mathrm{~nm}$. It has to be pointed out that these worst case crosstalks may not be found by simple measurements because of the requirement of precise polarization conditions along the fiber. Although the probability for these worst cases to happen is small, they have to be considered in system design.

\section{REFERENCES}

[1] A. R. Chraplyvy, "Limitation on lightwave communications imposed by optical fiber nonlinearities," J. Lightwave Technol., vol. 8, pp. 1548-1557, Oct. 1990.

[2] J. Wang, X. Sun, and M. Zheng, "Effect of group velocity dispersion on stimulated Raman crosstalk in multichannel transmission systems," IEEE Photon. Technol. Lett., vol. 10, pp. 540-542, Apr. 1998.

[3] K.-P. Ho, "Statistical properties of stimulated Raman crosstalk in WDM systems," J. Lightwave Technol., vol. 18, pp. 915-921, Ki;u 2000.

[4] M. R. Phillips and D. M. Ott, "Crosstalk due to optical fiber nonlinearities in WDM CATV lightwave systems," J. Lightwave Technol., vol. 17, pp. 1782-1792, Oct. 1999.

[5] F. Coppinger, L. Chen, and D. Piehler, "Nonlinear Raman cross-talk in a video overlay passive optical network," in Proc. Optical Fiber Communication Conf., OFC'2003, Atlanta, GA, Mar. 23-28, 2003, pp. 285-286.

[6] D. Derickson, Ed., Fiber Optic Test and Measurement. Englewood Cliffs, NJ: Prentice Hall, 1998.

[7] (2002, August) Corning SMF-28 Fiber Specification. [Online] http://www.corning.com/opticalfiber/products_services/product_catalog/smf-28/

${ }^{1}$ ITU-T Recommendation G.983.3, video channel wavelength 1550-1560 $\mathrm{nm}$, data channel wavelength $1480-1500 \mathrm{~nm}$. Therefore, the widest channel spacing is $80 \mathrm{~nm}$. 